\title{
Guidelines for the Diagnosis and Management of Food Allergy in the United States: Summary of the NIAID- Sponsored Expert Panel Report
}

Primary Authors: Joshua A. Boyce, MD, ${ }^{a}$ Amal Assa'ad, MD, ${ }^{d}$ A. Wesley Burks, MD, ${ }^{e}$ Stacie M. Jones, MD, ${ }^{9}$

Hugh A. Sampson, MD, ${ }^{\text {h }}$ Robert A. Wood, MD, ${ }^{i}$ Marshall Plaut, MD, ${ }^{j}$ Susan F. Cooper, MSc, ${ }^{j}$ and Matthew J. Fenton, PhD 
2.2.2. Prevalence of allergy to specific foods, foodinduced anaphylaxis, and food allergy with comorbid conditions 1109

\section{SECTION 3. NATURAL HISTORY OF FOOD ALLERGY AND ASSOCIATED DISORDERS 1110}

3.1. Natural history of food allergy in children 1110

3.2. Natural history of levels of allergen-specific $\operatorname{IgE}$ to foods in children 1110

3.3. Natural history of food allergy in adults 1110

3.4. Natural history of conditions that coexist with food allergy 1110

3.4.1. Asthma 1110

3.4.2. Atopic dermatitis 1110

3.4.3. Eosinophilic esophagitis 1110

3.4.4. Exercise-induced anaphylaxis 1110

3.5. Risk factors for the development of food allergy 1110

3.6. Risk factors for severity of allergic reactions to foods 1110

3.7. Incidence, prevalence, and consequences of unintentional exposure to food allergens 1110

J. A. Boyce has served on the Advisory Board of GlaxoSmithKline. He has served as a consultant and/or speaker for Altana, GlaxoSmithKline, and Merck. He has received funding/grant support from the National Institutes of Health.

A. Assa'ad holds, or is listed as an inventor on, US patent application \#10/566903, entitled "Genetic markers of food allergy." She has served as a consultant for GlaxoSmithKline and as a speaker for the American College of Allergy, Asthma, and Immunology, the North East Allergy Society, the Virginia Allergy Society, the New England Allergy Society, and the American Academy of Pediatrics. Dr Assa'ad has received funding/grant support from GlaxoSmithKline.

A. W. Burks holds, or is listed as an inventor on, multiple US patents related to food allergy. He owns stock in Allertein and MastCell, Inc, and is a minority stockholder in Dannon Co Probiotics. He has served as a consultant for ActoGeniX NV, McNeil Nutritionals, Mead Johnson, and Novartis. He has served on the speaker's bureau for EpiPen/Dey, LP, and has served on the data monitoring committee for Genentech. He has served on an expert panel for Nutricia. Dr Burks has received funding/grant support from the Food Allergy and Anaphylaxis Network, Gerber, Mead Johnson, and the National Institutes of Health.

S. M. Jones has served as a speaker and grant reviewer and has served on the medical advisory committee for the Food Allergy and Anaphylaxis Network. She has received funding/grant support from Dyax Corp, the Food Allergy and Anaphylaxis Network, Mead Johnson, the National Peanut Board, and the National Institutes of Health.

H. A. Sampson holds, or is listed as an inventor on, multiple US patents related to food allergy. He owns stock in Allertein Therapeutics. He is the immediate past president of the American Academy of Allergy, Asthma, and Immunology. He has served as a consultant for Allertein Therapeutics, the American Academy of Allergy, Asthma, and Immunology, the Food Allergy Initiative, and Schering Plough. He has received funding/grant support for research projects from the Food Allergy Initiative, the National Institutes of Health (Division of Receipt and Referral, National Institute of Allergy and Infectious Diseases, National Center for Complementary and Alternative Medicine), and Phadia AB. He is a co-owner of Herbal Spring, LLC.

R. A. Wood has served as a speaker/advisory board member for GlaxoSmithKline, Merck, and Dey. He has received funding/grant support from Genentech and the National Institutes of Health (National Institute of Allergy and Infectious Diseases).

S. H. Arshad has received funding/grant support from the National Institutes of Health and the National Institute of Health Research, UK.

S. L. Bahna has received funding/grant support from Genentech.

L. A. Beck has received funding/grant support from the American Academy of Allergy, Asthma, and Immunology, the National Eczema Association, and the National Institutes of Health.

C. Byrd-Bredbenner owns stock in Johnson \& Johnson. She has received funding/grant support from the US Department of Agriculture, the Canned Food Alliance, and the New Jersey Department of Health and Senior Services.

C. A. Camargo Jr has consulted for Dey and Novartis. He has received funding/grant support from a variety of government agencies and not-for-profit research foundations, as well as Dey and Novartis.

L. Eichenfield has received funding/grant support from a variety of not-for-profit foundations, as well as Astellas, Ferndale, Johnson \& Johnson, Novartis, Sinclair, Stiefel, and Therapeutics Inc.
SECTION 4. DIAGNOSIS OF FOOD ALLERGY 1110

4.1. When should food allergy be suspected? 1111

4.2. Diagnosis of IgE-mediated food allergy 1111

4.2.1. Medical history and physical examination 1111

4.2.2. Methods to identify the causative food 1111

4.2.2.1. Skin prick test 1111

4.2.2.2. Intradermal tests 1111

4.2.2.3. Total serum IgE 1111

4.2.2.4. Allergen-specific serum IgE 1111

4.2.2.5. Atopy patch test 1111

4.2.2.6. Use of skin prick tests, $\operatorname{sIgE}$ tests, and atopy patch tests in combination 1111

4.2.2.7. Food elimination diets 1111

4.2.2.8. Oral food challenges 1111

4.2.2.9. Nonstandardized and unproven procedures 1112

4.3. Diagnosis of non-IgE-mediated immunologic adverse reactions to food 1112

4.3.1. Eosinophilic gastrointestinal diseases 1112

4.3.2. Food protein-induced enterocolitis syndrome 1112 4.3.3. Food protein-induced allergic proctocolitis 1112

G. T. Furuta has served as a consultant and/or speaker to Ception Therapeutics and TAP He has received funding/grant support from the American Gastrointestinal Association and the National Institutes of Health.

J. M. Hanifin has served as served as a consultant for ALZA, Anesiva, Inc, Barrier Therapeutics, Inc, Milliken \& Company, Nordic Biotech, Novartis Pharmaceuticals Corporation, Shionogi USA, Taisho Pharmaceutical R\&D, Inc, Teikoku Pharma USA, Inc, UCB, York Pharma, ZARS, Inc, and ZymoGenetics. He has served as an investigator or received research funding from ALZA, Astellas Pharma US, Inc, Asubio Pharmaceuticals, Inc, Centocor, Inc, Corgentech, Novartis, Nucryst Pharmaceuticals, Seattle Genetics, and Shionogi USA.

M. Kraft has served as a consultant and/or speaker for Astra-Zeneca, Genentech, GlaxoSmithKline, Merck, Novartis, and Sepracor. She has received funding/grant support from Genentech, GlaxoSmithKline, the National Institutes of Health and Novartis.

B. D. Levy holds, or is listed as an inventor on, US patent applications \#20080064746 entitled "Lipoxins and aspirin-triggered lipoxins and their stable analogs in the treatment of asthma and inflammatory airway diseases" and \#20080096961 entitled "Use of docosatrienes, resolvins and their stable analogs in the treatment of airway diseases and asthma." He owns stock in Resolvyx Pharmaceuticals. He has served as a consultant for Bayer Healthcare and Resolvyx Pharmaceuticals. Dr Levy has received funding/grant support from the National Institutes of Health.

P. Lieberman has served as a consultant and/or speaker to Dey Laboratories, Novartis, Schering-Plough, AstraZenica, Merck, TEVA, Pfizer, MEDA, Alcon, Genentech, Intelliject, and the Food Allergy and Anaphylaxis Network. He is past president of the American Academy of Allergy, Asthma, and Immunology.

L. C. Schneider has served as a consultant/clinical advisor for the Food Allergy Initiative. She has received funding/grant support from a variety of not-for-profit research foundations, as well as Novartis and the National Institutes of Health.

R. A. Simon has served as a speaker for Dey Laboratories, Genentech, GlaxoSmithKline, Merck, Novartis, and the US Food and Drug Administration.

F. E. R. Simons holds a patent on "Fast-disintegrating epinephrine tablets for sublingual administration." She is a past-president of the American Academy of Allergy, Asthma and Immunology and of the Canadian Society of Allergy and Clinical Immunology. She is a member of the advisory boards of Dey, Intelliject, and ALK-Abello. She has received funding/grant support from AllerGen, the Canadian Allergy, Asthma and Immunology Foundation/Anaphylaxis Canada, and the Canadian Institutes of Health Research

S. J. Teach has served as a speaker for AstraZeneca. He has received funding/grant support from the AstraZeneca Foundation, Aventis, the Child Health Center Board, the CNMC Research Advisory Council, the National Association of Chain Drug Stores Foundation, the National Institutes of Health (National Institute of Allergy and Infectious Diseases; National Heart, Lung, and Blood Institute), Novartis/Genentech, the Robert Woods Johnson Foundation, the US Centers for Disease Control and Prevention, the US Public Health Service, and the Washington, DC, Department of Health The other authors have declared that they have no conflict of interest. 
4.3.4. Food protein-induced enteropathy syndrome 1112

4.3.5. Allergic contact dermatitis 1112

4.3.6. Systemic contact dermatitis 1112

4.4. Diagnosis of IgE-mediated contact urticaria 1112

\section{SECTION 5. MANAGEMENT OF NONACUTE ALLER- GIC REACTIONS AND PREVENTION OF FOOD ALLERGY 1112}

5.1. Management of individuals with food allergy 1112

5.1.1. Dietary avoidance of specific allergens in IgEmediated food allergy 1112

5.1.2. Dietary avoidance of specific allergens in nonIgE-mediated food allergy 1112

5.1.3. Effects of dietary avoidance on associated and comorbid conditions, such as atopic dermatitis, asthma, and eosinophilic esophagitis 1112

5.1.4. Food avoidance and nutritional status 1112

5.1.5. Food labeling in food allergy management 1112

5.1.6. When to re-evaluate patients with food allergy 1112

5.1.7. Pharmacologic intervention for the prevention of food-induced allergic reactions 1112

5.1.7.1. IgE-mediated reactions 1112

5.1.7.2. Non-IgE-mediated reactions 1112

5.1.8. Pharmacologic intervention for the treatment of food-induced allergic reactions 1113

5.1.9. Immunotherapy for food allergy management 1113

5.1.9.1. Allergen-specific immunotherapy 1113

5.1.9.2. Immunotherapy with cross-reactive allergens 1113

5.1.10. Quality-of-life issues associated with food allergy 1113

5.1.11. Vaccinations in patients with egg allergy 1113

5.1.11.1. Measles, mumps, rubella, and varicella vaccine 1113

5.1.11.2. Influenza vaccine 1113

5.1.11.3. Yellow fever vaccine 1113

5.1.11.4. Rabies vaccines 1113

5.2. Management of individuals at risk for food allergy 1113

5.2.1. Nonfood allergen avoidance in at-risk patients 1113

5.2.2. Dietary avoidance of foods with cross-reactivities in at-risk patients 1113

5.2.3. Testing of allergenic foods in patients at high risk prior to introduction 1113

5.2.4. Testing in infants and children with persistent atopic dermatitis 1114

5.3. Prevention of food allergy 1114

5.3.1. Maternal diet during pregnancy and lactation 1114

5.3.2. Breast-feeding 1114

5.3.3. Special diets in infants and young children 1114

5.3.3.1. Soy infant formula versus cow's milk formula 1114

5.3.3.2. Hydrolyzed infant formulas versus cow's milk formula or breast-feeding 1114

5.3.4. Timing of introduction of allergenic foods to infants 1114

SECTION 6. DIAGNOSIS AND MANAGEMENT OF FOOD-INDUCED ANAPHYLAXIS AND OTHER ACUTE ALLERGIC REACTIONS TO FOODS 1114

6.1. Definition of anaphylaxis 1114

6.2. Diagnosis of acute, life-threatening, food-induced allergic reactions 1114
6.3. Treatment of acute, life-threatening, food-induced allergic reactions 1114

6.3.1. First-line and adjuvant treatment for food-induced anaphylaxis 1114

6.3.2. Treatment of refractory anaphylaxis 1116

6.3.3. Possible risks of acute therapy for anaphylaxis 1116

6.3.4. Treatment to prevent biphasic or protracted food-induced allergic reactions 1116

6.3.5. Management of milder, acute food-induced allergic reactions in health care settings 1116

6.4. Management of food-induced anaphylaxis 1116

\section{APPENDIX A. PRIMARY AUTHOR AFFILIATIONS AND ACKNOWLEDGMENTS 1116}

\section{APPENDIX B. LIST OF ABBREVIATIONS 1118}

*Some numbered sections found in the Guidelines are not included in this Summary because they do not include a guideline recommendation or an "In summary" statement, or because the detailed information in the section was not suitable for a concise Summary.

\section{SECTION 1. INTRODUCTION}

\subsection{Overview}

Food allergy (FA) is an important public health problem that affects adults and children and may be increasing in prevalence. Despite the risk of severe allergic reactions and even death, there is no current treatment for FA: the disease can only be managed by allergen avoidance or treatment of symptoms. Moreover, the diagnosis of FA may be problematic, given that nonallergic food reactions, such as food intolerance, are frequently confused with FAs. Additional concerns relate to the differences in the diagnosis and management of FA in different clinical practice settings.

Due to these concerns, the National Institute of Allergy and Infectious Diseases (NIAID), part of the National Institutes of Health, working with more than 30 professional organizations, federal agencies, and patient advocacy groups, led the development of "best practice" clinical guidelines for the diagnosis and management of FA (henceforth referred to as the Guidelines). ${ }^{1}$ Based on a comprehensive review and objective evaluation of the recent scientific and clinical literature on FA, the Guidelines were developed by and designed for allergists/immunologists, clinical researchers, and practitioners in the areas of pediatrics, family medicine, internal medicine, dermatology, gastroenterology, emergency medicine, pulmonary and critical care medicine, and others.

The Guidelines focus on diseases that are defined as FA (see section 2.1) and include both IgE-mediated reactions to food and some non-IgE-mediated reactions to food. The Guidelines do not discuss celiac disease, which is an immunologic non- $\operatorname{IgE}-$ mediated reaction to certain foods. Although this is an immunebased disease involving food, existing clinical guidelines for celiac disease will not be restated here.

Finally, these Guidelines do not address the management of patients with FA outside of clinical care settings (for example, schools and restaurants) or the related public health policy issues. These issues are beyond the scope of this document.

\subsection{Relationship of the US Guidelines to other guidelines}

(Not summarized here; refer to Guidelines.) 
1.3. How the Guidelines were developed

1.3.1. The Coordinating Committee. NIAID established a Coordinating Committee (CC), whose members are listed in Appendix A of the Guidelines, to oversee the development of the Guidelines; review drafts of the Guidelines for accuracy, practicality, clarity, and broad utility of the recommendations in clinical practice; review the final Guidelines; and disseminate the Guidelines. The CC members were from 34 professional organizations, advocacy groups, and federal agencies, and each member was vetted for financial conflict of interest (COI) by NIAID staff.

1.3.2. The Expert Panel. The CC convened an Expert Panel (EP) in March 2009 that was chaired by Joshua Boyce, MD (Brigham and Women's Hospital, Boston, Mass). Panel members were specialists from a variety of relevant clinical, scientific, and public health areas (see Acknowledgments). Each member was vetted for financial COI by NIAID staff and approved by the $\mathrm{CC}$. The charge to the EP was to use an independent, systematic literature review, in conjunction with consensus expert opinion and EP-identified supplementary documents, to develop Guidelines that provide a comprehensive approach for diagnosing and managing FA based on the current state of the science.

1.3.3. The independent, systematic literature review and report. RAND Corporation prepared an independent, systematic literature review and evidence report on the state of the science in FA. This work was supported by an NIAID contract awarded in September 2008. The contract's principal investigator was Paul G. Shekelle, MD, PhD, an internationally recognized expert in the fields of practice guidelines and meta-analysis. RAND screened more than 12,300 titles, reviewed more than 1,200 articles, abstracted nearly 900 articles, and included 348 articles in the final RAND report. The full version of the report with a complete list of references is available at http://www.rand. org/pubs/working_papers/WR757-1/.

1.3.4. Assessing the quality of the body of evidence. In addition to assessing the quality of each of the included studies, RAND assessed the quality of the body of evidence using the Grading of Recommendations Assessment, Development and Evaluation (GRADE) approach, which was developed in 2004. GRADE provides a comprehensive and transparent methodology to develop recommendations for the diagnosis, treatment, and management of patients. In assessing the body of evidence, GRADE considers study design and other factors, such as the precision, consistency, and directness of the data. Using this approach, GRADE then provides a grade of high, moderate, or low for the quality of the body of evidence.

1.3.5. Preparation of the draft Guidelines and Expert Panel deliberations. All 43 clinical recommendations drafted by the EP received $90 \%$ (or higher) agreement. Sections 3, 5, and 6 of the Guidelines also contain "In summary" statements. These statements are intended to provide health care professionals with significant information that did not warrant a recommendation, or are in place of a recommendation when the $\mathrm{EP}$ or the $\mathrm{CC}$ could not reach consensus. All "In summary" statements received $90 \%$ (or higher) agreement.

1.3.6. Public comment period and draft Guidelines revision. The draft Guidelines were posted to the NIAID Web site in March 2010 for a period of 60 days to allow for public review and comment. More than 550 comments were collected and reviewed by the $\mathrm{CC}$, the EP, and NIAID. The EP revised the Guidelines in response to some of these comments. The final Guidelines were published in the December 2010 issue of the Journal of Allergy and Clinical Immunology and are publically available at www.jacionline.org.

\subsection{Defining the strength of each clinical guideline}

The EP has used the verb "recommends" or "suggests" in each clinical guideline. These words convey the strength of the guideline, defined as follows:

- Recommend is used when the EP strongly recommended for or against a particular course of action.

- Suggest is used when the EP weakly recommended for or against a particular course of action.

\subsection{Summary}

The Guidelines are intended to assist health care professionals in making appropriate decisions about patient care in the United States. The recommendations are not fixed protocols that must be followed. Health care professionals should take these Guidelines into account when exercising their clinical judgment. However, this guidance does not override their responsibility to make decisions appropriate to the circumstances of the individual patient, in consultation with the patient, guardian, or caregiver. Clinical judgment on the management of individual patients remains paramount. Health care professionals, patients, and their families need to develop individual treatment plans that are tailored to the specific needs and circumstances of the patient. This document is intended as a resource to guide clinical practice and develop educational materials for patients, their families, and the public. It is not an official regulatory document of any government agency.

\section{SECTION 2. DEFINITIONS, PREVALENCE, AND EPIDEMIOLOGY OF FOOD ALLERGY}

\subsection{Definitions}

2.1.1. Definitions of food allergy, food, and food allergens. The EP came to consensus on definitions used throughout the Guidelines.

A food allergy is defined as an adverse health effect arising from a specific immune response that occurs reproducibly on exposure to a given food.

A food is defined as any substance-whether processed, semiprocessed, or raw-that is intended for human consumption, and includes drinks, chewing gum, food additives, and dietary supplements. Substances used only as drugs, tobacco products, and cosmetics (such as lip-care products) that may be ingested are not included.

Food allergens are defined as those specific components of food or ingredients within food (typically proteins, but sometimes also chemical haptens) that are recognized by allergenspecific immune cells and elicit specific immunologic reactions, resulting in characteristic symptoms. Some allergens (most often from fruits and vegetables) cause allergic reactions primarily if eaten when raw. However, most food allergens can still cause reactions even after they have been cooked or have undergone digestion in the stomach and intestines. A phenomenon called 
cross-reactivity may occur when an antibody reacts not only with the original allergen, but also with a similar allergen. In FA, cross-reactivity occurs when a food allergen shares structural or sequence similarity with a different food allergen or aeroallergen, which may then trigger an adverse reaction similar to that triggered by the original food allergen. Cross-reactivity is common, for example, among different shellfish and different tree nuts.

Food oils—-such as soy, corn, peanut, and sesame—range from very low allergenicity (if virtually all of the food protein is removed in processing) to very high allergenicity (if little of the food protein is removed in processing).

2.1.2. Definitions of related terms. Because individuals can develop allergic sensitization (as evidenced by the presence of allergen-specific $\operatorname{IgE}(\mathrm{s} \operatorname{IgE})$ ) to food allergens without having clinical symptoms on exposure to those foods, an sIgEmediated FA requires both the presence of sensitization and the development of specific signs and symptoms on exposure to that food. Sensitization alone is not sufficient to define FA.

These Guidelines generally use the term tolerate to denote a condition where an individual has either naturally outgrown an FA or has received therapy and no longer develops clinical symptoms following ingestion of the food. This ability to tolerate food does not distinguish between these 2 possible clinical states. Individuals may tolerate food only for a short term, perhaps because they have been desensitized by exposure to the food. Alternatively, they may develop long-term tolerance. The specific term tolerance is used in these Guidelines to mean that an individual is symptom free after consumption of the food or upon oral food challenge weeks, months, or even years after the cessation of treatment. The immunological mechanisms that underlie tolerance in humans are poorly understood.

Although many different foods and food components have been recognized as food allergens, these Guidelines focus on only those foods that are responsible for the majority of observed adverse allergic or immunologic reactions. Moreover, foods or food components that elicit reproducible adverse reactions but do not have established or likely immunologic mechanisms are not considered food allergens. Instead, these non-immunologic adverse reactions are termed food intolerances. For example, an individual may be allergic to cow's milk (henceforth referred to as milk) due to an immunologic response to milk protein, or alternatively, that individual may be intolerant to milk due to an inability to digest the sugar lactose. In the former situation, milk protein is considered an allergen because it triggers an adverse immunologic reaction. Inability to digest lactose leads to excess fluid production in the gastrointestinal (GI) tract, resulting in abdominal pain and diarrhea. This condition is termed lactose intolerance, and lactose is not an allergen because the response is not immune based. It should be noted that the words tolerance and intolerance are unrelated terms, even though the spelling of the words implies that they are opposites.

2.1.3. Definitions of specific food-induced allergic conditions. The reader is referred to the Guidelines for the definitions of the following:

- Food-induced anaphylaxis

- GI food allergies and several specific syndromes

- Immediate GI hypersensitivity

- Eosinophilic esophagitis (EoE)
- Eosinophilic gastroenteritis

- Food protein-induced allergic proctocolitis (AP)

- Food protein-induced enterocolitis syndrome (FPIES)

- Oral allergy syndrome (OAS)

- Cutaneous reactions to foods

- Acute urticaria

- Angioedema

- The increase in atopic dermatitis (AD) symptoms

- Allergic contact dermatitis

- Contact urticaria

- Respiratory manifestations

- Heiner syndrome

\subsection{Prevalence and epidemiology of food allergy}

The true prevalence of FA has been difficult to establish for several reasons.

- Although more than 170 foods have been reported to cause IgE-mediated reactions, most prevalence studies have focused on only the most common foods.

- The incidence and prevalence of FA may have changed over time, and many studies have indeed suggested a true rise in prevalence over the past 10 to 20 years.

- Studies of FA incidence, prevalence, and natural history are difficult to compare because of inconsistencies and deficiencies in study design and variations in the definition of FA.

2.2.1. Systematic reviews of the prevalence of food allergy. (Not summarized here; refer to Guidelines.)

2.2.2. Prevalence of allergy to specific foods, foodinduced anaphylaxis, and food allergy with comorbid conditions. The following is a summary of prevalence data for the most common food allergies and anaphylaxis:

\section{Peanut}

- Prevalence of peanut allergy in the United States is about $0.6 \%$ of the population.

- Prevalence of peanut allergy in France, Germany, Israel, Sweden, and the United Kingdom varies between $0.06 \%$ and $5.9 \%$.

\section{Tree nuts}

- Prevalence of tree nut allergy in the United States is $0.4 \%$ to $0.5 \%$ of the population.

- Prevalence of tree nut allergy in France, Germany, Israel, Sweden, and the United Kingdom varies between $0.03 \%$ and $8.5 \%$.

\section{Seafood}

- Prevalence rates in the United States are significantly lower for children than for adults: fish allergy, $0.2 \%$ for children vs $0.5 \%$ for adults; crustacean shellfish allergy, $0.5 \%$ vs $2.5 \%$; any seafood allergy, $0.6 \%$ vs $2.8 \%$.

- Prevalence rates in the United States are higher for women than for men: crustacean shellfish allergy, $2.6 \%$ for women vs $1.5 \%$ for men; any fish, $0.6 \%$ vs $0.2 \%$.

\section{Milk and hen's egg}

- In a Danish cohort, allergy to milk was confirmed in $2.2 \%$. Of these, 54\% had IgE-mediated allergy, and the remaining $46 \%$ were classified as non-IgE mediated.

- In a Norwegian cohort, the prevalence of hen's egg (henceforth referred to as egg) allergy was estimated to be $1.6 \%$, and most egg reactions were IgE mediated. 
Food-induced anaphylaxis

- Several studies in the United States assessed the incidence of anaphylaxis related to food. These studies found wide differences in the rates (from 1/100,000 population to as high as 70/100,000 population) of hospitalization or emergency department visits for anaphylaxis, as assessed by International Classification of Diseases, Ninth Revision, Clinical Modification (ICD-9-CM) codes or medical record review.

- The proportion of anaphylaxis cases thought to be due to foods also varied between $13 \%$ and $65 \%$, with the lowest percentages found in studies that used more stringent diagnostic criteria for anaphylaxis.

The EP agreed that any estimate of the overall US incidence of anaphylaxis is unlikely to have utility because such an estimate fails to reflect the substantial variability in patient age, geographic distribution, criteria used to diagnose anaphylaxis, and the study methods used.

\section{SECTION 3. NATURAL HISTORY OF FOOD ALLERGY AND ASSOCIATED DISORDERS}

The EP reviewed the literature on the natural history of FA and summarized the available data for the most common food allergens in the United States: egg, milk, peanut, tree nuts, wheat, crustacean shellfish, and soy. Natural history data for fish allergy were unavailable as of the completion of the systematic literature review (September 2009). It should be noted that many published studies addressing the natural history of FA typically come from selected populations (for example, from a single clinic or hospital) that may not be representative of the general or community-based patient population with a specific FA condition. Thus, the findings of these studies may not necessarily be extrapolated to all patients with the condition.

\subsection{Natural history of food allergy in children}

In summary: Most children with FA eventually will tolerate milk, egg, soy, and wheat; far fewer will eventually tolerate tree nuts and peanut. The time course of FA resolution in children varies by food and may occur as late as the teenage years. A high initial level of sIgE against a food is associated with a lower rate of resolution of clinical allergy over time.

\subsection{Natural history of levels of allergen-specific $\lg E$ to foods in children}

In summary: For many patients, sIgE antibodies to foods appear within the first 2 years of life. Levels may increase or decrease; a decrease is often associated with the ability to tolerate the foods.

\subsection{Natural history of food allergy in adults}

In summary: FA in adults can reflect persistence of pediatric FAs (for example, milk, peanut, and tree nuts) or de novo sensitization to food allergens encountered after childhood. Although there is a paucity of data from US studies, FA that starts in adult life tends to persist.
3.4. Natural history of conditions that coexist with food allergy

In summary: $\mathrm{FA}$ may coexist with asthma, $\mathrm{AD}, \mathrm{EoE}$, and exercise-induced anaphylaxis. In patients with asthma, the coexistence of FA may be a risk factor for severe asthma exacerbations. Moreover, food may be a trigger for exercise-induced anaphylaxis. Elimination of food allergens in sensitized individuals can improve symptoms of some comorbid conditions.

\subsubsection{Asthma.}

In summary: Asthma and FA often coexist in pediatric and adult patients. FA is associated with severe asthma.

\subsubsection{Atopic dermatitis.}

In summary: AD and FA are highly associated. When tolerance develops to a food, the reintroduction of the food in the diet will not result in recurrence or worsening of the AD.

\subsubsection{Eosinophilic esophagitis.}

In summary: EoE is commonly associated with sensitization to foods. The natural history of EoE is that of a chronic condition that resolves spontaneously or with therapy, and then relapses. There are insufficient data to judge the impact of food sensitization on the natural history of EoE, and vice versa. Only retrospective data exist that support a beneficial effect of dietary changes on the histopathologic changes in the esophagus in EoE.

\subsubsection{Exercise-induced anaphylaxis.}

In summary: Exercise-induced anaphylaxis in adults is triggered by foods in about one third of patients and has a natural history marked by frequent recurrence of the episodes.

\subsection{Risk factors for the development of food allergy}

In summary: Family history of atopy and the presence of AD are risk factors for the development of both sensitization to food and confirmed FA.

\subsection{Risk factors for severity of allergic reactions to foods}

In summary: The severity of allergic reactions to foods is multifactorial and variable. The severity of a reaction cannot be accurately predicted by the degree of severity of past reactions nor by the level of $\operatorname{sgE}$ or the size of the wheal from the skin prick test (SPT). The factor most commonly identified with the most severe reactions is the coexistence of asthma.

\subsection{Incidence, prevalence, and consequences of unintentional exposure to food allergens}

In summary: Self-reported reactions to food frequently occur in patients with a known diagnosis of FA. Although a subset of these reactions is due to intentional exposure, most are due to unintentional exposure. Both types of exposure can be lifethreatening. There is no evidence that unintentional or intentional exposures to the food allergen alter the natural history of the FA.

\section{SECTION 4. DIAGNOSIS OF FOOD ALLERGY}

\subsection{When should food allergy be suspected?}

Guideline 1: The EP recommends that FA should be considered: 
TABLE I. Symptoms of food-induced allergic reactions

\begin{tabular}{|c|c|c|}
\hline Target organ & Immediate symptoms & Delayed symptoms \\
\hline Cutaneous & $\begin{array}{l}\text { Erythema } \\
\text { Pruritus } \\
\text { Urticaria } \\
\text { Morbilliform eruption } \\
\text { Angioedema }\end{array}$ & $\begin{array}{l}\text { Erythema } \\
\text { Flushing } \\
\text { Pruritus } \\
\text { Morbilliform eruption } \\
\text { Angioedema } \\
\text { Eczematous rash }\end{array}$ \\
\hline Ocular & $\begin{array}{l}\text { Pruritus } \\
\text { Conjunctival erythema } \\
\text { Tearing } \\
\text { Periorbital edema }\end{array}$ & $\begin{array}{l}\text { Pruritus } \\
\text { Conjunctival erythema } \\
\text { Tearing } \\
\text { Periorbital edema }\end{array}$ \\
\hline $\begin{array}{l}\text { Upper } \\
\text { respiratory }\end{array}$ & $\begin{array}{l}\text { Nasal congestion } \\
\text { Pruritus } \\
\text { Rhinorrhea } \\
\text { Sneezing } \\
\text { Laryngeal edema } \\
\text { Hoarseness } \\
\text { Dry staccato cough }\end{array}$ & \\
\hline $\begin{array}{l}\text { Lower } \\
\quad \text { respiratory }\end{array}$ & $\begin{array}{l}\text { Cough } \\
\text { Chest tightness } \\
\text { Dyspnea } \\
\text { Wheezing } \\
\text { Intercostal retractions } \\
\text { Accessory muscle use }\end{array}$ & $\begin{array}{l}\text { Cough, dyspnea, } \\
\text { and wheezing }\end{array}$ \\
\hline GI (oral) & $\begin{array}{l}\text { Angioedema of the lips, } \\
\text { tongue, or palate } \\
\text { Oral pruritus } \\
\text { Tongue swelling }\end{array}$ & \\
\hline GI (lower) & $\begin{array}{l}\text { Nausea } \\
\text { Colicky abdominal pain } \\
\text { Reflux } \\
\text { Vomiting } \\
\text { Diarrhea }\end{array}$ & $\begin{array}{l}\text { Nausea } \\
\text { Abdominal pain } \\
\text { Reflux } \\
\text { Vomiting } \\
\text { Diarrhea } \\
\text { Hematochezia } \\
\text { Irritability and } \\
\text { food refusal with } \\
\text { weight loss (young } \\
\text { children) }\end{array}$ \\
\hline Cardiovascular & $\begin{array}{l}\text { Tachycardia (occasionally } \\
\text { bradycardia in anaphylaxis) } \\
\text { Hypotension } \\
\text { Dizziness } \\
\text { Fainting } \\
\text { Loss of consciousness }\end{array}$ & \\
\hline Miscellaneous & $\begin{array}{l}\text { Uterine contractions } \\
\text { Sense of "impending } \\
\text { doom" }\end{array}$ & \\
\hline
\end{tabular}

GI, Gastrointestinal.

Note: This table is presented as Table IV in the Guidelines.

- In individuals presenting with anaphylaxis or any combination of symptoms listed in Table I that occur within minutes to hours of ingesting food, especially in young children and/or if symptoms have followed the ingestion of a specific food on more than 1 occasion

- In infants, young children, and selected older children diagnosed with certain disorders, such as moderate to severe $\mathrm{AD}$, EoE, enterocolitis, enteropathy, and allergic proctocolitis

- In adults diagnosed with EoE
4.2. Diagnosis of IgE-mediated food allergy

4.2.1. Medical history and physical examination.

Guideline 2: The EP recommends using medical history and physical examination to aid in the diagnosis of FA.

- Medical history: The EP recommends using a detailed medical history to help focus the evaluation of an FA. Although the medical history often provides evidence for the type of food-induced allergic reaction and the potential causative food(s) involved, history alone cannot be considered diagnostic of FA.

- Physical examination: The EP recommends performing a focused physical examination of the patient, which may provide signs consistent with an allergic reaction or disorder often associated with FA. However, by itself, the physical examination cannot be considered diagnostic of FA.

Guideline 3: The EP recommends that parent and patient reports of FA must be confirmed, because multiple studies demonstrate that $50 \%$ to $90 \%$ of presumed FAs are not allergies.

\subsubsection{Methods to identify the causative food.}

\subsubsection{Skin prick test.}

Guideline 4: The EP recommends performing an SPT (also known as a skin puncture test) to assist in the identification of foods that may be provoking IgE-mediated food-induced allergic reactions, but the SPT alone cannot be considered diagnostic of FA.

\subsubsection{Intradermal tests.}

Guideline 5: The EP recommends that intradermal testing should not be used to make a diagnosis of FA.

\subsubsection{Total serum IgE.}

Guideline 6: The EP recommends that the routine use of measuring total serum IgE should not be used to make a diagnosis of FA.

\subsubsection{Allergen-specific serum IgE.}

Guideline 7: The EP recommends SIgE tests for identifying foods that potentially provoke IgE-mediated food-induced allergic reactions, but alone these tests are not diagnostic of FA.

\subsubsection{Atopy patch test.}

Guideline 8: The EP suggests that the atopy patch test (APT) should not be used in the routine evaluation of non-contact FA.

\subsubsection{Use of skin prick tests, slgE tests, and atopy patch tests in combination.}

Guideline 9: The EP suggests not using the combination of SPTs, sIgE tests, and APTs for the routine diagnosis of FA.

\subsubsection{Food elimination diets.}

Guideline 10: The EP suggests that elimination of 1 or a few specific foods from the diet may be useful in the diagnosis of FA, especially in identifying foods responsible for some non-IgE-mediated food-induced allergic disorders, such as FPIES, AP, and Heiner syndrome, and some mixed IgE- and non-IgE-mediated food-induced allergic disorders, such as EoE.

\subsubsection{Oral food challenges.}

Guideline 11: The EP recommends using oral food challenges for diagnosing FA. The double-blind placebo-controlled food challenge is the gold standard. However, a single-blind or an open-food challenge may be considered diagnostic under certain circumstances: if either of these challenges elicits no symptoms (ie, the challenge is negative), then FA can be ruled out; but when either challenge elicits objective symptoms (ie, the challenge is positive) and those objective symptoms correlate with 
medical history and are supported by laboratory tests, then a diagnosis of FA is supported.

\subsubsection{Nonstandardized and unproven procedures.}

Guideline 12: The EP recommends not using any of the following nonstandardized tests for the routine evaluation of IgE-mediated FA:

- Basophil histamine release/activation

- Lymphocyte stimulation

- Facial thermography

- Gastric juice analysis

- Endoscopic allergen provocation

- Hair analysis

- Applied kinesiology

- Provocation neutralization

- Allergen-specific $\mathrm{IgG}_{4}$

- Cytotoxicity assays

- Electrodermal test (Vega)

- Mediator release assay (LEAP diet)

\subsection{Diagnosis of non-IgE-mediated immunologic ad-} verse reactions to food.

\subsubsection{Eosinophilic gastrointestinal diseases.}

Guideline 13: The EP suggests that SPTs, sIgE tests, and APTs may be considered to help identify foods that are associated with EoE, but these tests alone are not sufficient to make the diagnosis of FA. The role of these tests in the diagnosis of other eosinophilic GI disorders has not been established.

4.3.2. Food protein-induced enterocolitis syndrome.

Guideline 14: The EP recommends using the medical history and oral food challenge to establish a diagnosis of FPIES. However, when history indicates that infants or children have experienced hypotensive episodes or multiple reactions to the same food, a diagnosis may be based on a convincing history and absence of symptoms when the causative food is eliminated from the diet.

\subsubsection{Food protein-induced allergic proctocolitis.}

Guideline 15: The EP recommends using the medical history, resolution of symptoms when the causative food is eliminated from the diet, and recurrence of symptoms following an oral food challenge to diagnose allergic proctocolitis.

4.3.4. Food protein-induced enteropathy syndrome. (Not summarized here; refer to Guidelines.)

\subsubsection{Allergic contact dermatitis.}

Guideline 16: The EP recommends using the medical history, including the absence of symptoms while the causative food is avoided, and positive patch tests to diagnose allergic contact dermatitis.

\subsubsection{Systemic contact dermatitis.}

Guideline 17: The EP suggests using the medical history, including the resolution of symptoms while the causative food is avoided, and positive patch tests to establish the diagnosis of systemic contact dermatitis.

\subsection{Diagnosis of IgE-mediated contact urticaria}

Guideline 18: The EP suggests using the medical history, including the absence of symptoms while the causative food is avoided, positive sIgE tests or SPTs, and positive immediate epicutaneous skin tests (for example, positive immediate responses to APTs), to establish the diagnosis of food-induced IgEmediated contact urticaria.
Contact urticaria can be of 2 types, either IgE mediated or nonIgE mediated. In IgE-mediated contact urticaria, substances present in foods interact with sIgE bound to cutaneous mast cells, leading to the release of histamine and other inflammatory mediators. Localized or generalized urticaria, as well as systemic symptoms, may result. sIgE-mediated contact urticaria may be assessed with APTs, SPTs, or sIgE tests, although there is no standardization of diagnostic methodology. In non-IgE-mediated contact urticaria to food, systemic symptoms are rarely seen.

\section{SECTION 5. MANAGEMENT OF NONACUTE ALLERGIC REACTIONS AND PREVENTION OF FOOD ALLERGY}

\subsection{Management of individuals with food allergy 5.1.1. Dietary avoidance of specific allergens in lgE- mediated food allergy.}

Guideline 19: The EP recommends that individuals with documented IgE-mediated FA should avoid ingesting their specific allergen or allergens.

\subsubsection{Dietary avoidance of specific allergens in non- IgE-mediated food allergy.}

Guideline 20: The EP recommends that individuals with documented non-IgE-mediated FA should avoid ingesting their specific allergen or allergens.

5.1.3. Effects of dietary avoidance on associated and comorbid conditions, such as atopic dermatitis, asthma, and eosinophilic esophagitis.

Guideline 21: In individuals with documented or proven FA who also have 1 or more of the following - $\mathrm{AD}$, asthma, or EoE-the EP recommends avoidance of their specific allergen or allergens.

Guideline 22: In individuals without documented or proven FA, the EP does not recommend avoiding potentially allergenic foods as a means of managing AD, asthma, or EoE.

\subsubsection{Food avoidance and nutritional status.}

Guideline 23: The EP recommends nutritional counseling and regular growth monitoring for all children with FA.

\subsubsection{Food labeling in food allergy management.}

Guideline 24: The EP suggests that individuals with FA and their caregivers receive education and training on how to interpret ingredient lists on food labels and how to recognize labeling of the food allergens used as ingredients in foods. The EP also suggests that products with precautionary labeling, such as "this product may contain trace amounts of allergen," be avoided.

5.1.6. When to re-evaluate patients with food allergy.

Guideline 25: The EP suggests follow-up testing for individuals with FA depending on the specific food to which the individual is allergic. Whether testing is done annually or at other intervals depends on the food in question, the age of the child, and the intervening medical history.

5.1.7. Pharmacologic intervention for the prevention of food-induced allergic reactions.

\subsubsection{IgE-mediated reactions.}

Guideline 26: There are no medications currently recommended by the EP to prevent IgE-mediated food-induced allergic reactions from occurring in an individual with existing FA.

\subsubsection{Non-IgE-mediated reactions.}

Guideline 27: There are no medications currently recommended by the EP to prevent non-IgE-mediated food-induced allergic reactions from occurring in an individual with existing FA. 
5.1.8. Pharmacologic intervention for the treatment of food-induced allergic reactions. Allergen avoidance is the first line of treatment for FA, and use of antihistamines, as needed, remains the mainstay of managing (as opposed to preventing) symptoms of nonsevere food-induced allergic reactions. However, drug therapy has been used to treat FA in cases where allergen avoidance is extremely difficult or results in nutritional deficiencies. Drugs that alter the immune response to the allergen are commonly considered the most likely candidates for such therapy in the future, but these treatments are not currently recommended (see Guideline 28).

\subsubsection{Immunotherapy for food allergy management.}

\subsubsection{Allergen-specific immunotherapy.}

Guideline 28: The EP does not recommend using allergenspecific immunotherapy to treat IgE-mediated FA.

\subsubsection{Immunotherapy with cross-reactive allergens.}

Guideline 29: The EP does not recommend immunotherapy with cross-reactive allergens for treating IgE-mediated FA.

\subsubsection{Quality-of-life issues associated with food} allergy.

Guideline 30: The EP recommends that patients with FA and their caregivers be provided with information on food allergen avoidance and emergency management that is age and culturally appropriate.

\subsubsection{Vaccinations in patients with egg allergy.}

In summary: Patients who have generated IgE antibodies to an allergen are at risk for anaphylaxis with systemic exposure to that allergen. Thus, patients who have IgE-mediated egg allergy are at risk for anaphylaxis if injected with vaccines containing egg protein. More detailed information about specific egg-containing vaccines (MMR, MMRV, influenza, yellow fever, and rabies) is provided in sections 5.1.11.1-5.1.11.4 of the Guidelines.

The EP recognizes that changes in these recommendations may occur in the future as there is an increased understanding of the risk factors for allergic reactions and as vaccine manufacturing processes improve and decrease the final egg protein content of vaccines. For the most current recommendations, health care professionals should refer to the Web sites of the American Academy of Pediatrics (AAP) and Advisory Committee for Immunization Practices (ACIP):

- http://aapredbook.aappublications.org/

- http://www.cdc.gov/vaccines/recs/acip/

\subsubsection{Measles, mumps, rubella, and varicella vaccine.}

Guideline 31: The EP recognizes the varying consensus recommendations of the different organizations on this particular vaccine and recommends that children with egg allergy, even those with a history of severe reactions, receive vaccines for measles, mumps, and rubella (MMR) and for MMR with varicella (MMRV). The safety of this practice has been recognized by ACIP and AAP and is noted in the approved product prescribing information for these vaccines.

\subsubsection{Influenza vaccine.}

In summary: The EP concludes that insufficient evidence exists to recommend administering influenza vaccine, either inactivated or live-attenuated, to patients with a history of severe reactions to egg proteins. Severe reactions include a history of hives, angioedema, allergic asthma, or systemic anaphylaxis to egg proteins (or chicken proteins). Less severe or local manifestations of allergy to egg or feathers are not contraindications. However, the EP notes that egg allergy is relatively common among the very patients who would highly benefit from influenza vaccination. Such patients include children and young adults (from 6 months to 18 years old for seasonal influenza, and from 6 months to 24 years old for H1N1 influenza) and all patients with asthma. It should be noted that live-attenuated vaccine is not licensed for use in patients with asthma.

Although ACIP and AAP, and also the vaccine manufacturers, do not recommend influenza vaccination in patients who are allergic to egg, several publications have described different approaches to giving the influenza vaccine to patients with severe allergic reactions to egg. These approaches, which depend on the ovalbumin content and the results of SPTs or intradermal tests with the vaccine, include a single dose of vaccine, 2 doses of vaccine, or multiple doses. However, the evidence supporting these approaches is limited by the small numbers of patients included in each study. Moreover, data indicate that, although the vaccines are relatively safe, there remains some, albeit low, risk of systemic reactions. Also, negative SPT results do not accurately predict safety of vaccination, in that $5 \%$ of patients with negative SPTs had systemic reactions to vaccination.

\subsubsection{Yellow fever vaccine.}

In summary: The EP recognizes the current guidelines from the different organizations and recommends against administering yellow fever vaccine to patients with a history of hives, angioedema, allergic asthma, or systemic anaphylaxis to egg proteins, unless an allergy evaluation and testing with the vaccine is done first. This approach has been recognized by ACIP and AAP and is noted in the approved product prescribing information for this vaccine.

\subsubsection{Rabies vaccines.}

In summary: The EP recognizes the current guidelines from the different organizations and recommends against administering rabies vaccines to patients with a history of hives, angioedema, allergic asthma, or systemic anaphylaxis to egg proteins, unless an allergy evaluation and testing with the vaccine is done first. This approach has been recognized by ACIP and AAP and is noted in the approved product prescribing information for these vaccines.

\subsection{Management of individuals at risk for food allergy}

5.2.1. Nonfood allergen avoidance in at-risk patients.

Guideline 32: The EP suggests that patients at risk for developing FA do not limit exposure to potential nonfood allergens (for example, dust mites, pollen, or pet dander). Patients at risk for developing FA are defined as those with a biological parent or sibling with existing, or history of, allergic rhinitis, asthma, AD, or FA. This definition of "at risk" is used throughout sections 5.2 and 5.3. 5.2.2. Dietary avoidance of foods with crossreactivities in at-risk patients.

Guideline 33: The EP suggests that patients at risk for developing FA do not need to limit exposure to foods that may be cross-reactive with the 8 major food allergens in the United States (milk, egg, peanut, tree nuts, soy, wheat, fish, and crustacean shellfish).

\subsubsection{Testing of allergenic foods in patients at high} risk prior to introduction.

In summary: The EP concludes that insufficient evidence exists to recommend routine FA testing prior to the introduction of highly allergenic foods (such as milk, egg, and peanut) in children 
who are at high risk of reacting to the introduction of such foods. The definition of children at high risk, in this specific situation, is children with pre-existing severe allergic disease and/or a family history of FA. Nevertheless, there may be some value in FA evaluations that include an oral food challenge for a select group of patients with certain risk factors, such as having a sibling with peanut allergy or evidence of another underlying FA (for example, testing for tree nut allergy in a child with peanut allergy). It is possible that an FA evaluation prior to introduction of a food could potentially prevent allergic reactions. However, widespread SPTs and sIgE tests are not recommended because of their poor predictive value. These tests would lead to many clinically irrelevant results and unnecessary dietary restrictions, especially if unconfirmed by oral food challenges. Overall, the risk-to-benefit ratio of FA evaluation should be considered on an individual basis, especially for the highly allergenic foods in high-risk young children.

Guideline 34: The EP suggests that the general population of children not be tested for FA to highly allergenic foods prior to their introduction into the diet. The general population of children does not have pre-existing severe allergic disease and also does not have a family history of FA.

5.2.4. Testing in infants and children with persistent atopic dermatitis.

Guideline 35: The EP suggests that children less than 5 years old with moderate to severe AD be considered for FA evaluation for milk, egg, peanut, wheat, and soy, if at least 1 of the following conditions is met:

- The child has persistent AD in spite of optimized management and topical therapy.

- The child has a reliable history of an immediate reaction after ingestion of a specific food.

\subsection{Prevention of food allergy}

\subsubsection{Maternal diet during pregnancy and lactation.}

Guideline 36: The EP does not recommend restricting maternal diet during pregnancy or lactation as a strategy for preventing the development or clinical course of FA.

\subsubsection{Breast-feeding.}

Guideline 37: The EP recommends that all infants be exclusively breast-fed until 4 to 6 months of age, unless breastfeeding is contraindicated for medical reasons.

\subsubsection{Special diets in infants and young children.}

5.3.3.1. Soy infant formula versus cow's milk formula.

Guideline 38: The EP does not recommend using soy infant formula instead of cow's milk infant formula as a strategy for preventing the development of FA or modifying its clinical course in at-risk infants ( "at risk" is defined in Guideline 32).

\subsubsection{Hydrolyzed infant formulas versus cow's milk formula or breast-feeding.}

Guideline 39: The EP suggests that the use of hydrolyzed infant formulas, as opposed to cow's milk formula, may be considered as a strategy for preventing the development of FA in at-risk infants who are not exclusively breast-fed ( "at risk" is defined in Guideline 32). Cost and availability of extensively hydrolyzed infant formulas may be weighed as prohibitive factors.

\subsubsection{Timing of introduction of allergenic foods to} infants.

Guideline 40: The EP suggests that the introduction of solid foods should not be delayed beyond 4 to 6 months of age. Potentially allergenic foods may be introduced at this time as well.

\section{SECTION 6. DIAGNOSIS AND MANAGEMENT OF FOOD-INDUCED ANAPHYLAXIS AND OTHER ACUTE ALLERGIC REACTIONS TO FOODS}

\subsection{Definition of anaphylaxis}

Anaphylaxis is defined as a serious allergic reaction that is rapid in onset and may cause death. Typically, IgE-mediated food-induced anaphylaxis is believed to involve systemic mediator release from sensitized mast cells and basophils. The term anaphylactoid has been used in the past to indicate adverse reactions that are not IgE mediated and typically are not life-threatening. This term is imprecise and will not be used in these Guidelines.

\subsection{Diagnosis of acute, life-threatening, food- induced allergic reactions}

Guideline 41: The EP recommends that the health care professional considering a diagnosis of food-induced anaphylaxis should understand:

- The signs and symptoms characteristic of anaphylaxis

- The timing of symptoms in association with food ingestion/ exposure

- Comorbid conditions, such as asthma, that may affect treatment and outcome

- The limited utility of laboratory parameters in the acutecare setting

\subsection{Treatment of acute, life-threatening, food- induced allergic reactions}

6.3.1. First-line and adjuvant treatment for foodinduced anaphylaxis.

Guideline 42: The EP recommends that treatment for foodinduced anaphylaxis should focus on the following:

- Prompt and rapid treatment after onset of symptoms (see Table II)

- Intramuscular (IM) epinephrine as first-line therapy

- Other treatments, which are adjunctive to epinephrine dosing

Epinephrine is the first-line treatment in all cases of anaphylaxis. All other drugs have a delayed onset of action. When there is suboptimal response to the initial dose of epinephrine, or if symptoms progress, repeat epinephrine dosing remains first-line therapy over adjunctive treatments.

The cornerstones of initial management should begin with the following concurrent steps:

- Elimination of additional allergen exposure

- IM injection of epinephrine

- Call for help (summon a resuscitation team in the hospital setting, call 911 or an equivalent service in the community setting), although attempts to summon help should not delay use of epinephrine

These actions should be quickly followed by these additional steps:

- Placement of the patient in a recumbent position (if tolerated), with the lower extremities elevated

- Provision of supplemental oxygen

- Administration of intravenous (IV) fluid (volume resuscitation) 
TABLE II. Summary of the pharmacologic management of anaphylaxis (modified)

Note: These treatments often occur concomitantly, and are not meant to be sequential, with the exception of epinephrine as first-line treatment.

In the outpatient setting

- First-line treatment:

- Epinephrine, IM; auto-injector or 1:1,000 solution

O Weight 10 to $25 \mathrm{~kg}$ : $0.15 \mathrm{mg}$ epinephrine autoinjector, IM (anterior-lateral thigh)

○ Weight $>25 \mathrm{~kg}$ : $0.3 \mathrm{mg}$ epinephrine autoinjector, IM (anterior-lateral thigh)

Epinephrine (1:1,000 solution) (IM), $0.01 \mathrm{mg} / \mathrm{kg}$ per dose; maximum dose, $0.5 \mathrm{mg}$ per dose (anterior-lateral thigh)

-Epinephrine doses may need to be repeated every 5-15 minutes

-Adjunctive treatment:

-Bronchodilator ( $\beta_{2}$-agonist): albuterol

MDI (child: $4-8$ puffs; adult: 8 puffs) or

O Nebulized solution (child: $1.5 \mathrm{ml}$; adult: $3 \mathrm{ml}$ ) every 20 minutes or continuously as needed

$-\mathrm{H}_{1}$ antihistamine: diphenhydramine

O 1 to $2 \mathrm{mg} / \mathrm{kg}$ per dose

- Maximum dose, $50 \mathrm{mg}$ IV or oral (oral liquid is more readily absorbed than tablets)

○ Alternative dosing may be with a less-sedating second generation antihistamine

- Supplemental oxygen therapy

- IV fluids in large volumes if patient presents with orthostasis, hypotension, or incomplete response to IM epinephrine

- Place the patient in recumbent position if tolerated, with the lower extremities elevated

\section{In the hospital-based setting}

- First-line treatment:

- Epinephrine IM as above, consider continuous epinephrine infusion for persistent hypotension (ideally with continuous non-invasive monitoring of blood pressure and heart rate); alternatives are endotracheal or intra-osseous epinephrine

- Adjunctive treatment:

- Bronchodilator $\left(\beta_{2}\right.$-agonist): albuterol

MDI (child: 4-8 puffs; adult: 8 puffs) or

O Nebulized solution (child: $1.5 \mathrm{ml}$; adult: $3 \mathrm{ml}$ ) every 20 minutes or continuously as needed

- $\mathrm{H}_{1}$ antihistamine: diphenhydramine

○ 1 to $2 \mathrm{mg} / \mathrm{kg}$ per dose

O Maximum dose, $50 \mathrm{mg}$ IV or oral (oral liquid is more readily absorbed than tablets)

$\circ$ Alternative dosing may be with a less-sedating second generation antihistamine

- $\mathrm{H}_{2}$ antihistamine: ranitidine

○ 1 to $2 \mathrm{mg} / \mathrm{kg}$ per dose

O Maximum dose, 75 to $150 \mathrm{mg}$ oral and IV

- Corticosteroids

○ Prednisone at $1 \mathrm{mg} / \mathrm{kg}$ with a maximum dose of 60 to $80 \mathrm{mg}$ oral or

○ Methylprednisolone at $1 \mathrm{mg} / \mathrm{kg}$ with a maximum dose of 60 to $80 \mathrm{mg} \mathrm{IV}$

- Vasopressors (other than epinephrine) for refractory hypotension, titrate to effect

- Glucagon for refractory hypotension, titrate to effect

○ Child: $20-30 \mu \mathrm{g} / \mathrm{kg}$

O Adult: $1-5 \mathrm{mg}$

○ Dose may be repeated or followed by infusion of $5-15 \mu \mathrm{g} / \mathrm{min}$

- Atropine for bradycardia, titrate to effect

- Supplemental oxygen therapy

- IV fluids in large volumes if patients present with orthostasis, hypotension, or incomplete response to IM epinephrine

- Place the patient in recumbent position if tolerated, with the lower extremities elevated

\section{Therapy for the patient at discharge}

- First-line treatment:

- Epinephrine auto-injector prescription (2 doses) and instructions

- Education on avoidance of allergen

- Follow-up with primary care physician

- Consider referral to an allergist

- Adjunctive treatment:

- $\mathrm{H}_{1}$ antihistamine: diphenhydramine every 6 hours for 2-3 days; alternative dosing with a non-sedating second generation antihistamine

- $\mathrm{H}_{2}$ antihistamine: ranitidine twice daily for 2-3 days

- Corticosteroid: prednisone daily for 2-3 days 
In summary: The use of antihistamines is the most common reason reported for not using epinephrine and may place a patient at significantly increased risk for progression toward a lifethreatening reaction.

6.3.2. Treatment of refractory anaphylaxis. No published prospective studies exist on the optimal management of refractory anaphylactic shock. Repeated use of epinephrine, as well as IV fluids, corticosteroids, and vasopressor agents, may be needed. Prompt transfer to an acute-care facility and intensive-care unit for treatment and monitoring is essential.

6.3.3. Possible risks of acute therapy for anaphylaxis. There are no absolute contraindications to epinephrine use in anaphylaxis. However, there are subgroups of patients who might theoretically be at higher risk for adverse effects during epinephrine therapy. Because the risk of death or serious disability from anaphylaxis itself usually outweighs other concerns, existing evidence clearly favors the benefit of epinephrine administration in most situations. Some level of decision making regarding the risk-to-benefit ratio may be warranted for patients under specific circumstances (described in the Guidelines).

6.3.4. Treatment to prevent biphasic or protracted foodinduced allergic reactions. Very little information exists that defines the mechanism of biphasic or protracted allergic reactions. Similarly, little information exists to support specific therapy to prevent biphasic or protracted food-induced allergic reactions. In general, induction and recruitment of inflammatory cells and release of preformed, long-acting mediators from mast cells have been implicated as mechanisms. Due to their anti-inflammatory properties, systemic corticosteroids are often recommended to prevent biphasic or protracted food-induced allergic reactions, but little data support their use.

6.3.5. Management of milder, acute food-induced allergic reactions in health care settings. Milder forms of allergic reactions, such as flushing, urticaria, isolated mild angioedema, or symptoms of OAS, can be treated with $\mathrm{H}_{1}$ and $\mathrm{H}_{2}$ antihistamine medications. When antihistamines alone are given, ongoing observation and monitoring are warranted to ensure a lack of progression to more significant symptoms of anaphylaxis. If progression or increased severity is noted, epinephrine should be administered immediately. Additionally, if there is a history of a prior severe allergic reaction, epinephrine should be administered promptly and earlier in the course of treatment (for example, at the onset of even mild symptoms).

\subsection{Management of food-induced anaphylaxis}

Guideline 43: The EP recommends that the management of food-induced anaphylaxis should focus on the following:

- Dosing with IM epinephrine followed by transfer to an emergency facility for observation and possible further treatment

- Observation for 4 to 6 hours or longer based on severity of the reaction

- Education for patient and family on:

- Allergen avoidance

- Early recognition of signs and symptoms of anaphylaxis

- Anaphylaxis emergency action plan implementation

- Appropriate IM epinephrine administration

- Medical identification jewelry or an anaphylaxis wallet card

- Epinephrine auto-injector prescription and training provided at the time of discharge
- Continuation of adjunctive treatment after patient discharge:

- $\mathrm{H}_{1}$ antihistamine: diphenhydramine every 6 hours for 23 days; alternative dosing with a non-sedating second generation antihistamine

$-\mathrm{H}_{2}$ antihistamine: ranitidine twice daily for 2-3 days

- Corticosteroid: prednisone daily for 2-3 days

- Follow-up appointment with primary health care professional (after the food-induced anaphylactic reaction), with consideration for additional follow-up with a clinical specialist such as an allergist/immunologist

\section{REFERENCE}

1. Boyce JA, Assa'ad A, Burks WA, Jones SM, Sampson HA, Wood RA, et al. Guidelines for the Diagnosis and Management of Food Allergy in the United States: Report of the NIAID-Sponsored Expert Panel. J Allergy Clin Immunol 2010;126(suppl 1): S1-58.

\section{APPENDIX A. PRIMARY AUTHOR AFFILIATIONS AND ACKNOWLEDGMENTS}

Primary authors

*Joshua A. Boyce, MD

Division of Rheumatology, Immunology and Allergy

Brigham and Women's Hospital

Department of Medicine Harvard Medical School

Boston, Mass

*Amal Assa'ad, MD

Division of Allergy and Immunology

Cincinnati Children's Hospital Medical Center

University of Cincinnati

Cincinnati, Ohio

*A. Wesley Burks, MD

Division of Allergy and Immunology

Department of Pediatrics

Duke University Medical Center

Durham, NC

*Stacie M. Jones, MD

Division of Allergy and Immunology

Department of Pediatrics

University of Arkansas for Medical Sciences

Arkansas Children's Hospital

Little Rock, Ark

*Hugh A. Sampson, MD

Elliot and Roslyn Jaffe Food Allergy Institute

Division of Allergy and Immunology

Department of Pediatrics

Mount Sinai School of Medicine

New York, NY

*Robert A. Wood, MD

Division of Allergy and Immunology

Department of Pediatrics

The Johns Hopkins University School of Medicine

Baltimore, Md

Marshall Plaut, MD

Division of Allergy, Immunology, and Transplantation 
National Institute of Allergy and Infectious Diseases National Institutes of Health

Bethesda, Md

\section{Susan F. Cooper, MSc}

Division of Allergy, Immunology, and Transplantation

National Institute of Allergy and Infectious Diseases

National Institutes of Health

Bethesda, Md

\section{Matthew J. Fenton, PhD}

Division of Allergy, Immunology, and Transplantation

National Institute of Allergy and Infectious Diseases

National Institutes of Health

Bethesda, Md

Authors who are members of the Expert Panel are denoted with an asterisk $(*)$ in this list.

For additional information, please contact the National Institute of Allergy and Infectious Diseases at daitinfo@niaid.nih.gov.

\section{Acknowledgments}

\section{NIAID-sponsored Expert Panel}

\section{S. Hasan Arshad, MBBS, MRCP, DM, FRCP}

School of Medicine

University of Southampton

Southampton, UK

The David Hide Asthma and Allergy Research Centre

St. Mary's Hospital

Newport, Isle of Wight, UK

Southampton University Hospital NHS Trust

Southampton, UK

\section{Sami L. Bahna, MD, DrPH}

Department of Pediatrics

Allergy and Immunology Section

Louisiana State University Health Sciences Center

Shreveport, La

Lisa A. Beck, MD

Department of Dermatology

University of Rochester Medical Center

Rochester, NY

Carol Byrd-Bredbenner, PhD, RD, FADA

Department of Nutritional Sciences

Rutgers University

New Brunswick, NJ

\section{Carlos A. Camargo, Jr, MD, DrPH}

Department of Emergency Medicine and Division of Rheumatology, Allergy and Immunology

Department of Medicine

Massachusetts General Hospital

Harvard Medical School

Boston, Mass

\section{Lawrence Eichenfield, MD}

Division of Pediatric and Adolescent Dermatology

Rady Children's Hospital

San Diego, Calif

Departments of Pediatrics and Medicine
University of California, San Diego

San Diego, Calif

Glenn T. Furuta, MD

Section of Pediatric Gastroenterology, Hepatology, and Nutrition

Digestive Health Institute

Children's Hospital Denver

Aurora, Colo

Department of Pediatrics

National Jewish Health

Denver, Colo

Department of Pediatrics

University of Colorado Denver School of Medicine

Aurora, Colo

Jon M. Hanifin, MD

Department of Dermatology

Oregon Health and Science University

Portland, Ore

Carol Jones, RN, AE-C

Asthma Educator and Consultant

Allergy and Asthma Network

Mother's of Asthmatics

McLean, Va

\section{Monica Kraft, MD}

Division of Pulmonary, Allergy and Critical Care Medicine

Department of Medicine

Duke University Medical Center

Durham, NC

Bruce D. Levy, MD

Partners Asthma Center

Pulmonary and Critical Care Medicine Division

Brigham and Women's Hospital and Harvard Medical School

Boston, Mass

Phil Lieberman, MD

Division of Allergy and Immunology

Department of Medicine

University of Tennessee

College of Medicine

Memphis, Tenn

\section{Stefano Luccioli, MD}

Office of Food Additive Safety

US Food and Drug Administration

College Park, Md

Kathleen M. McCall, BSN, RN

Children's Hospital of Orange County

Orange, Calif

Lynda C. Schneider, MD

Division of Immunology

Children's Hospital Boston

Boston, Mass

\section{Ronald A. Simon, MD}

Division of Allergy, Asthma and Immunology

Scripps Clinic

San Diego, Calif 


\section{F. Estelle R. Simons, MD}

Departments of Pediatrics and Child Health and Immunology Faculty of Medicine

University of Manitoba

Winnipeg, Manitoba, Canada

\section{Stephen J. Teach, MD, MPH}

Division of Emergency Medicine

Children's National Medical Center

Washington, DC

\section{Barbara P. Yawn, MD, MPH, MSc}

Department of Research

Olmsted Medical Center

Rochester, Minn

Department of Family and Community Health

University of Minnesota

School of Medicine

Minneapolis, Minn

\section{NIAID staff}

\section{Julie M. Schwaninger, MSc}

Division of Allergy, Immunology, and Transplantation National Institute of Allergy and Infectious Diseases

National Institutes of Health

Bethesda, Md

\section{APPENDIX B. LIST OF ABBREVIATIONS}

AAP: American Academy of Pediatrics

ACIP: Advisory Committee on Immunization Practices

AD: Atopic dermatitis

AP: Allergic proctocolitis

APT: Atopy patch test

CC: Coordinating Committee

COI: Conflict of interest

EoE: Eosinophilic esophagitis

EP: Expert Panel

FA: Food allergy

FPIES: Food protein-induced enterocolitis syndrome

GI: Gastrointestinal

GRADE: Grading of Recommendations Assessment, Development and Evaluation

ICD-9-CM: International Classification of Diseases, Ninth Revision, Clinical Modification

IM: Intramuscular

IV: Intravenous

MDI: Metered-dose inhaler

MMR: Measles, mumps, and rubella

MMRV: Measles, mumps, rubella, and varicella

NIAID: National Institute of Allergy and Infectious Diseases

OAS: Oral allergy syndrome

sIgE: Allergen-specific IgE

SPT: Skin prick test 